\title{
P269: Infection control practices during labor and delivery and newborn care in resource limited settings: assessment and recommendations for improvement
}

\author{
WC Huskins ${ }^{1 *}, V$ Manchanda ${ }^{2}$, N Singh ${ }^{3}$ \\ From 2nd International Conference on Prevention and Infection Control (ICPIC 2013) \\ Geneva, Switzerland. 25-28 June 2013
}

\section{Introduction}

India is amongst the countries with high maternal mortality rate (MMR) \& infant mortality rate (IMR). Most of these deaths occur due to sepsis. UNICEF initiated a review of infection control practices (ICP) during labor \& delivery (L\&D) \& in newborn care at two states of Rajasthan \& Odisha, India.

\section{Objectives}

To assess current ICP during L\&D and newborn care with an aim to improve ICP to reduce MMR \& IMR due to infections.

\section{Methods}

A two member consultant team was constituted by UNICEF Delhi, India. The team conducted a structured assessment of different facilities (sub-district and district hospitals in Rajasthan \& Odisha) on ICP in L\&D rooms \& Special Care Newborn Units (SCNUs). The team completed the assessments using the Infection Control Assessment Tool $[1,2]$. Draft reports were provided to the respective offices on the evening of the assessments.

\section{Results}

Assessments were completed in 5 community health centers and district hospitals with L\&D rooms and SCNUs. Defined systems for cleanliness and general hygiene were conspicuously absent. Hand hygiene practices were poor due to lack of awareness \& supplies. Biological waste segregation was not appropriate and there were concerns regarding storage and disposal of bio-medical waste. Exposure risk to healthcare workers including doctors, nursing staff, and support staff is area of major concern.

\section{Conclusion}

The National Program has provided funds and brought enormous patient load to healthcare setting. However, training and uninterrupted supplies for L\&D and SCNU must be ensured. Specific recommended role of UNICEF and state health ministry of two states were highlighted.

\section{Competing interests}

None declared.

\section{Author details}

'Division of Pediatric Infectious Diseases, Mayo Clinic, Rochester, MN, USA. ${ }^{2}$ Clinical Microbiology and Infectious Diseases, Chacha Nehru Bal Chikitsalaya New Delhi, India. ${ }^{3}$ Division Infectious Diseases, Children's National Medical Center, Washington, DC, USA.

\section{Published: 20 June 2013}

\section{References}

1. Huskins WC, Ross-Degnan D, Goldmann DA: Improving infection control in developing countries: the Infection Control Assessment Tool. BMC ProC 2011, 5(Suppl 6):018.

2. Management Sciences for Health: Infection Control Assessment Tool., second 2009 [http://www.msh.org/projects/sps/SPS-Documents/upload/ ICAT-User-Manual_Global-May-09.pdf].

doi:10.1186/2047-2994-2-S1-P269

Cite this article as: Huskins et al:: P269: Infection control practices during labor and delivery and newborn care in resource limited settings: assessment and recommendations for improvement. Antimicrobial Resistance and Infection Control 2013 2(Suppl 1):P269. 